\title{
Correction
}

\section{Correction: Van Nuenen et al., Weight-Specific Anticipatory Coding of Grip Force in Human Dorsal Premotor Cortex}

In the article "Weight-Specific Anticipatory Coding of Grip Force in Human Dorsal Premotor Cortex" by Bart F.L. van Nuenen, Johann Kuhtz-Buschbeck, Christian Schulz, Bastiaan R. Bloem, and Hartwig R. Siebner, which appeared on pages 5272-5283 of the April 11, 2012 issue, the authors regret an error in Figure 4. The correct Figure 4 is shown below.

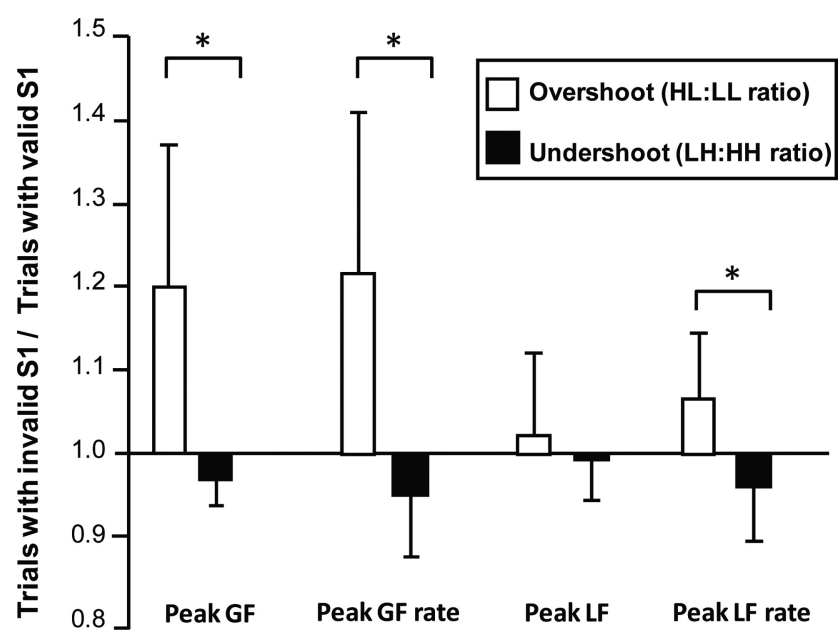

